\title{
MS-Medikation offenbar sicher
}

Das Risiko für einen schweren COVID-19-Verlauf bei Multipler Sklerose wird durch Immunmodulatoren nicht gesteigert. Weiters fragen sich Experten wo all die Schlaganfallpatienten bleiben.

Ende März hat die „MS international federation" (msif) unter Berufung auf renommierte MS-Experten Empfehlungen zur MS-Therapie während der COVID-19-Pandemie veröffentlicht. Darin wird für Basistherapeutika kein erhöhtes Risiko für einen schweren Verlauf vermutet, bei einer Lymphozyten-depletierenden Therapie jedoch zur besonderen Vorsicht geraten. Eine aktuelle Analyse scheint nun den eher geringen Einfluss von krankheitsmodifizierenden MS-Therapeutika (DMT) auf den Verlauf bei COVID-19 zu bestätigen. Neurologen um Dr. Céline Louapre von der Sorbonne in Paris haben Angaben des französischen COVID-19-Registers Covisep für MS-Patienten ausgewertet [1]. Sie konnten 347 MS-Kranke identifizieren, die zwischen Anfang März und Mitte Mai eine SARS-CoV-2-Infektion hatten. Im Schnitt waren die Patienten 45 Jahre alt und seit rund 14 Jahren an MS erkrankt, der mediane EDSS-Wert erreichte 2,0 Punkte, $19 \%$ hatten eine progrediente MS-Form (PMS).

\section{Schwerer Verlauf bei hohen EDSS-Werten, höherem Alter und Adipositas}

$18 \%$ der Erkrankten - primär die mit einer PMS - erhielten keine spezifischen MS-Medikamente, $35 \%$ wurden mit Basistherapeutika behandelt (Interferone, Glatirameracetat, Teriflunomid, Dimethylfumarat), für die kein oder ein gering erhöhtes Infektionsrisiko angenommen wird, die übrigen $47 \%$ nahmen stärker wirksame Therapeutika mit einem meist höheren systemischen Infektionsrisiko, hauptsächlich Natalizumab, Fingolimod und Ocrelizumab.

Jeder fünfte MS-Patient entwickelte einen schweren Verlauf, davon wurde bei einer Hospitalisierung ausgegangen. Zwölf MS-Kranke starben an der Infektion, das waren primär ältere Patienten mit einer PMS und hohen EDSS-Werten.

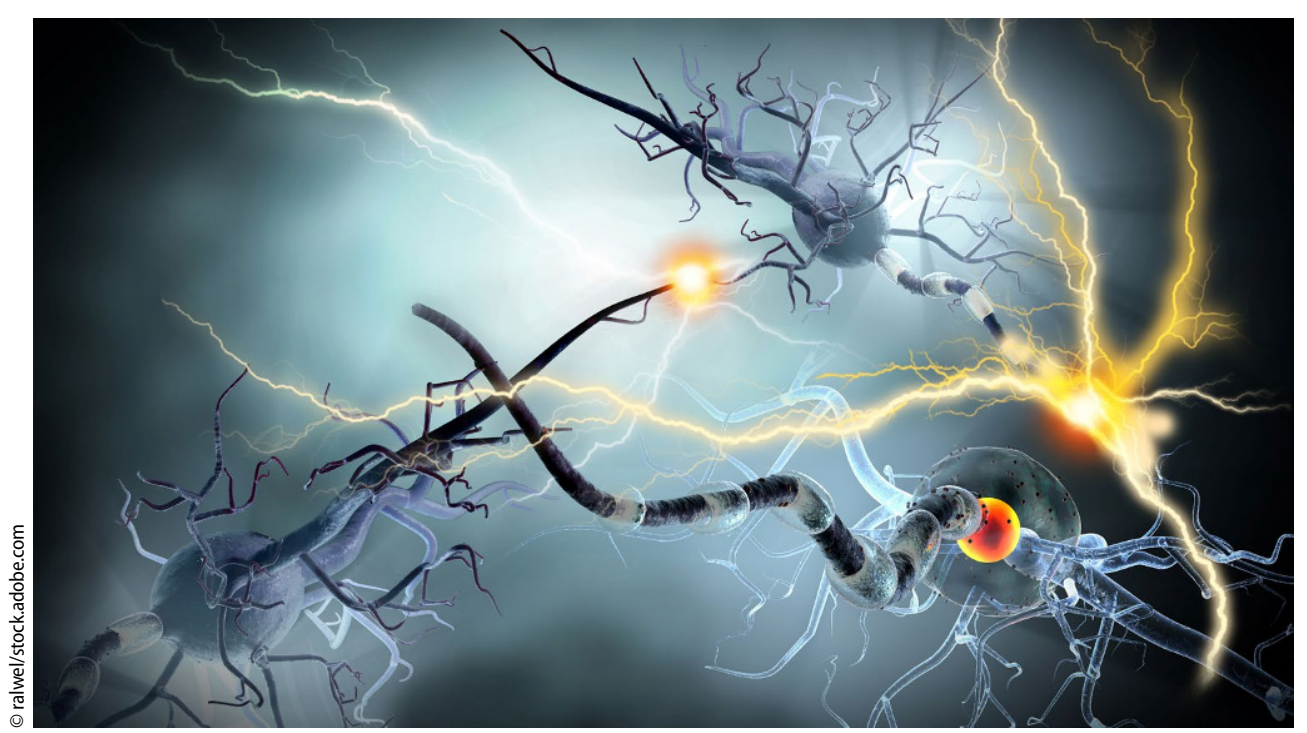

Schauten sich die Ärzte die Verteilung der unterschiedlichen Therapeutika bei Patienten mit schweren und weniger schweren Verläufen an, fanden sie durchaus Unterschiede: Nur drei der Patienten (6\%) unter Interferonen und Glatirameracetat entwickelten einen schweren COVID19-Verlauf, jedoch $13 \%$ unter den anderen Basistherapeutika sowie $24 \%$ mit den hochwirksamen DMT. Zudem zeigten sich die bekannten Risikofaktoren: Die hospitalisierten Patienten waren im Schnitt älter (55 versus 42 Jahre), hatten häufiger eine PMS (49 versus $11 \%$ ) und höhere EDSS-Werte $(6,0$ versus 2,0$)$, zudem waren sie öfter adipös (14 versus $5 \%$ ) als Patienten mit milderen Symptomen.

Berücksichtigen die Forscher in einer multivariaten Analyse sämtliche bekannten Begleitfaktoren, gingen jedoch nur noch ein hohes Alter, hohe EDSS-Werte und eine Adipositas mit einem schweren Verlauf einher, wobei der EDSS-Wert den stärksten Einfluss zeigte, gefolgt vom Alter. Das höchste Risiko für einen schweren COVID19-Verlauf haben demnach ältere Patienten mit einer stark fortgeschrittenen MS

》) Experten sehen keinen Grund, eine MS-Therapie aufgrund des COVID-19-Risikos zu verzögern

Die Neurologen um Louapre sehen daher keinen Grund, eine MS-Therapie aufgrund des COVID-19-Risikos zu verzögern oder die Therapie bei Infizierten abzubrechen. Letzteres wurde bei einem Teil der Patienten gemacht, dies hatte aber keinen Einfluss auf den Verlauf. 
Patienten mit leichten Schlaganfällen bleiben zuhause

Ein Rätsel ist für viele Neurologen noch immer das Fernbleiben der Schlaganfallpatienten: So ist das Schlaganfallrisiko bei COVID-19 erhöht, es müssten also eher mehr Schlaganfallpatienten in die Kliniken kommen, beobachtet wird jedoch das Gegenteil. Dafür gibt es zwei mögliche Erklärungen: Entweder ist die Schlaganfallinzidenz im Lockdown tatsächlich gesunken, etwa durch Faktoren wie weniger Feinstaub oder eine geringere Arbeitsbelastung, oder aber Schlaganfallpatienten gehen aus Angst vor einer Infektion seltener in eine Klinik - dies dürften dann wohl eher die leichten Fälle sein, spekulieren Neurologen um Dr. Richard Perry vom University College in London (UCL).

Sie verglichen daher einmal die Schwere der Schlaganfälle in den Wochen vor und während des Lock- downs. Wie sie vermutet hatten, kamen während des Lockdowns praktisch ebenso viele Patienten mit einem schweren Schlaganfall (NIHSSWert über 8) in die Klinik wie zuvor, aber dramatisch weniger mit einem leichten Insult - ihr Anteil schrumpfte zeitweise um fast $80 \%$.

》) Ärzte gehen davon aus, dass tatsächlich viele Patienten mit leichteren Schlaganfällen zu Hause geblieben sind

Da die meisten Schlaganfalltrigger und -risikofaktoren keinen Einfluss auf die Schwere des Ereignisses haben, gehen die Ärzte um Perry davon aus, dass die Inzidenz nicht wirklich gesunken ist, sondern tatsächlich viele Patienten mit leichteren Schlaganfällen zu Hause geblieben sind. Weil rund $10 \%$ solcher Patienten ohne Behandlung innerhalb einer Woche einen weiteren Insult ent- wickelten, sei dies jedoch keine gute Idee. Das Risiko für eine schwere SARSCoV-2-Infektion in einer Klinik sei da weitaus geringer [2].

\section{Literatur}

1. Louapre C et al (2020) Clinical Characteristics and Outcomes in Patients With Coronavirus Disease 2019 and Multiple Sclerosis. JAMA Neurol. https://doi.org/10.1001/jamaneurol.2020.2581

2. Perry R et al (2020) What has caused the fall in stroke admissions during the COVID-19 pandemic? J Neurol. https:// doi.org/10.1007/s00415-020-10030-2

Hinweis des Verlags. Der Verlag bleibt in Hinblick auf geografische Zuordnungen und Gebietsbezeichnungen in veröffentlichten Karten und Institutsadressen neutral.

psychopraxis.neuropraxis2020-23:166-167 https://doi.org/10.1007/s00739-02000663-3

(C) Springer-Verlag GmbH Austria, ein Teil von Springer Nature 2020
Quelle: www.springermedizin.de, Autor: Thomas Müller

Hier steht eine Anzeige. 